\title{
Robust Complex-Time Distributions based on Reconstruction Algorithms
}

\author{
Srdjan Stanković, Irena Orović \\ Faculty of Electrical Engineering, University of Montenegro \\ Podgorica, Montenegro \\ e-mail: irenao@ac.me
}

\begin{abstract}
The complex-time distributions has been used as an important time-frequency tool for the analysis of signals characterized by the fast-varying instantaneous frequency. However, in the presence of impulse noise, the distribution performance is seriously degraded, even in the case when the robust statistics (such as L-estimation) is applied instead of standard distribution calculation. As an efficient solution, we propose a combination of compressive sensing reconstruction and time-frequency distribution, which will be referred as compressive sensing based complex-time distribution.
\end{abstract}

Keywords-complex-lag distributions; compressive sensing; impulse noise; sparse signals

\section{INTRODUCTION}

Time-frequency analysis, as a very attractive research area, has been intensively developed during the last few decades. As a result, various time-frequency distributions have been proposed for the analysis of different non-stationary signals [1]-[3]. They are used in numerous practical applications in the areas of biomedical signal analysis [4], radar signals [5],[6] communications, geoscience, multimedia signals processing and applications [7]-[10], hardware implementations [11], etc. Time-frequency distributions are generally classified into: linear (spectrogram), quadratic (the Wigner and Cohen class distributions), and higher order distributions (polynomial and complex-time distributions) [12],[13]. It is important to emphasize that the higher order time-frequency distributions have been introduced for non-linear frequency modulated signals (higher order phase nonlinearity) to improve the concentration and precision of instantaneous frequency estimation, in comparison with quadratic distributions.

Particularly, the complex-time distributions were introduced to provide the efficient analysis of signals with highly non-stationary and fast-varying instantaneous frequency [13]-[23]. During the last decade, different forms of complextime distributions have been intensively studied by many researchers. Moreover, they have been used in different applications dealing with real world signals [19],[20], showing improved performance compared to other distributions. It is also worth to mention that both the hardware and software implementations of complex-time distribution have been successfully done [18], [21], allowing real-time processing. However, the complex-lag distributions, as well as other higher order distributions, are much more sensitive to noise which often occurs in real applications [24]. Namely, since the local auto-correlation function is obtained as a product of several noisy signal terms, the resulting noise will have impulsive nature. In this case the standard form of complex-time distributions does not to produce successful time-frequency representation and IF estimation. Thus, in the presence of noise, instead of the standard complex-lag distributions, we might use robust approaches such as the L-estimation based form [25][26]. The L-estimation concept is based on the alpha trimmed filter which can be efficient in noisy environment with an unknown noise probability density function. This approach assumes discarding the signal samples corrupted by impulse noise. However, due to the missing samples, the resulting timefrequency representation will be spoiled by new noisy effects. Therefore, in order to provide an efficient complex-time distribution, there is a need to recover the missing samples that are discarded to eliminate impulse noise. Nowadays, a very popular concept used in sparse signal reconstruction is known as compressive sensing, [24], [27]. Namely, it assumes that a signal which is sparse in certain transform domain can be randomly sampled in another (dense) domain, taking much lower number of samples than required by the sampling theorem. Then, using the optimization algorithms, the entire signal information can be recovered. In our case, the signal will not be compressively sampled, but the local complex-lag autocorrelation function will miss certain random samples discarded due to the noise. Thus, the idea is to employ the optimization algorithms to recover the samples of the autocorrelation function, and to obtain almost an ideal complextime distribution.

The paper is organized as follows. The theoretical background on the complex-lag distributions is given in Section II. The compressive sensing reconstruction of the local complex-lag autocorrelation function is proposed in Section III. The experimental evaluation is provided in Section IV, while the concluding remarks are given in Section V.

\section{THEORY}

In the case of signals with highly non-stationary phase function, the concentration in the time-frequency domain depends on the rate of IF variations, as well as on the distribution order and form. Hence, the complex-lag

This work is supported by the Montenegrin Ministry of Science. 
distributions have been introduced [13]-[15] to deal with signals whose instantaneous frequency varies fast, even within a few samples. The $N$-th order time-frequency distribution with complex-lag argument has been defined as [16]:

$$
C T D_{N}(t, \omega)=\int_{-\infty}^{\infty} \prod_{i=1}^{N / 2} \aleph\left(t, \tau, a_{i}, b_{i}\right) e^{-j \omega \tau} d \tau
$$

where,

$$
\aleph\left(t, \tau, a_{i}, b_{i}\right)=x\left(t+\frac{\tau}{N\left(a_{i}+j b_{i}\right)}\right)^{\left(a_{i}+j b_{i}\right)} x\left(t-\frac{\tau}{N\left(a_{i}+j b_{i}\right)}\right)^{-\left(a_{i}+j b_{i}\right)}
$$

The distribution order is an even number denoted by $N$. In practical applications, the signal with complex-lag argument is calculated by using the signal with real argument as follows:

$$
x\left(t \pm\left(a_{i}+j b_{i}\right) \tau\right)=\frac{1}{2 \pi} \int_{-\infty}^{\infty} X(\omega) e^{j \omega\left(t \pm\left(a_{i}+j b_{i}\right) \tau\right)} d \omega,
$$

where $X(\omega)$ is the Fourier transform of $x(t)$. The parameters $a_{i}$ and $b_{i}$ define the symmetrical complex points on the unit circle [16]. The presence of signal terms with symmetrical points $\pm\left(a_{i}+j b_{i}\right)$ eliminates all even phase derivatives from the spread factor. By a suitable choice of distribution order, some odd phase derivatives can be removed as well.

As one of the most interesting and commonly used cases of complex-lag distributions, let us observe the distribution defined by the following set of parameters:

$$
N=4, a_{1}=1, b_{1}=0, a_{2}=0, b_{2}=1 \text {. }
$$

The corresponding form of the complex-lag distribution is given by the following relation:

$$
C T D_{4}(t, \omega)=\int_{-\infty}^{\infty} x\left(t+\frac{\tau}{4}\right) x^{-1}\left(t-\frac{\tau}{4}\right) x^{-j}\left(t+j \frac{\tau}{4}\right) x^{j}\left(t-j \frac{\tau}{4}\right) e^{-j \omega \tau} d \tau
$$

the spread factor is obtained as:

$$
Q(t, \tau)=\phi^{(5)}(t) \frac{\tau^{5}}{4^{4} 5 !}+\phi^{(9)}(t) \frac{\tau^{9}}{4^{8} 9 !}+\phi^{(11)}(t) \frac{\tau^{11}}{4^{10} 11 !}+\ldots
$$

Note that, the dominant term in the spread factor is of the fifth order which assures an ideal concentration for signals with polynomial phase up to the fourth order. Therefore, the distribution (3) provides significant concentration improvement with respect to the quadratic distributions, where the spread factor contains even the third phase derivative, but also improvements compared to the polynomial distribution (of the same order $N=4$ ).

The discrete form of the complex-lag distribution (3) which is used in practical applications is given by:

$$
C T D_{4}(n, k)=\sum_{m=-N_{s} / 2}^{N_{s} / 2-1} R(n, m) e^{-j \frac{2 \pi}{N_{s}} 4 m k},
$$

where $R(n, m)$ denotes the complex-lag autocorrelation function:

$$
R(n, m)=w(m) x(n+m) x^{-1}(n-m) x^{-j}(n+j m) x^{j}(n-j m),
$$

parameters $n, k$ and $m$ denotes discrete time, frequency and lag coordinate, respectively, while $N_{s}$ is the number of samples within the window $w$.

\section{COMPRESSIVE SENSING L-ESTIMATE COMPLEX-TIME DISTRIBUTIONS}

In the case of noisy signals, the local complex-lag autocorrelation function $R(n, m)$ will be affected by an impulsive noise even when the signal is corrupted with Gaussian noise only. Thus to provide an efficient analysis in the presence of impulse noise, we apply the L-statistics for each $n=n_{i}$ to define the L-estimate higher order distributions:

$$
\operatorname{LCTD}\left(n_{i}, k\right)=\sum_{l=1}^{N s} p_{l} \operatorname{LCTD}_{(l)}\left(n_{i}, k\right), \quad,
$$

$\operatorname{LCTD}_{(l)} \in L C T D=\operatorname{sort}\left\{R\left(n_{i}, m\right) e^{-j 2 \pi m k / N s}, m=-N_{s} / 2, \ldots, N_{s} / 2-1\right\}$.

Sorting is done in non-decreasing order, while the coefficients are given by:

$$
p_{l}=N s-\alpha(N s-2), l \in[1, N s-\alpha(N s-2)],
$$

while $p_{l}$ is zero otherwise. Parameter $\alpha$ takes values within the range $[0,1]$ and defines the amount of samples that will be discarded. More samples we discard, stronger degradation we introduce in the resulting complex-time distribution.

In other words, we actually start from the fact that the Lestimation applied to time-frequency distribution removes impulses, but may cause other serious drawback by introducing another noise due to the discarded samples. The new compressive sensing concept provides the possibility to recover the missing samples and to avoid this side effect. Thus, in the sequel we show that the performance of the common L-estimation approach can be significantly improved by adopting CS reconstruction algorithms [28].

In CS, the signal, which is sparse in certain transform domain, can be reconstructed from a small set of measurements by using convex optimization algorithms. The sparse signal to be recovered can be sparse in its own domain or in some of the transform domains (DFT, DWT, DCT, etc). In general, a signal which is $K$ sparse in a specific domain can be completely characterized by $M$ measurements $(M>K)$ with $M<<N$, where $N$ is the number of samples imposed by the Shanon-Nyquist theorem. In that sense, the remaining samples are used for CS reconstruction of autocorrelation function $R$, which will lead to the CS based complex-time distribution. 


\section{A. Compressive sensing approach to complex-lag distribution}

Let us observe a discrete-time data set $R$ of length $N_{s}$ (in our case $R$ is the local complex-lag autocorrelation function). Any signal can be represented in terms of basis vectors as follows [27]:

$$
R=\sum_{i=1}^{N} \Re_{i} \psi_{i}=\psi \Re,
$$

where $\mathfrak{R}_{i}$ represents the transform domain coefficient, $\psi_{i}$ is a basis vector, $\psi$ denotes $N_{s} \mathrm{x} N_{s}$ transform matrix whose columns are basis vectors. If only $K$ transform coefficients from $\Re$ have non-zero values, we can say that $R$ is $\mathrm{K}$-sparse in transform domain defined by $\psi$.

Signal measurements belong to the domain where signal have "dense" representation. Despite the dimensionality reduction $\left(M<N_{s}\right)$, information needed to recover signal is well preserved, if the procedure satisfies certain conditions. Firstly, the measurement matrix $\phi$ must be incoherent with the basis matrix $\psi$. The coherence between two matrices measures the largest correlation between any two elements of matrices and it is defined as follows:

$$
\mu(\phi, \psi)=\sqrt{N s} \max _{k \geq 1, j \leq N s}\left|\left\langle\phi_{k}, \psi_{j}\right\rangle\right|,
$$

where $\phi_{k}$ and $\psi_{j}$ are row vector and column vector of the $\phi$ and $\psi$ matrices. The coherence has values in the range:

$$
1 \leq \mu(\phi, \psi) \leq \sqrt{N s},
$$

and its value increases as the elements of two matrices are more correlated. Lower coherence between $\phi$ and $\psi$ leads to a smaller number of measurements required to recover the entire signal. This number can be estimated as follows:

$$
M \geq c K \log (N s / K),
$$

where $c$ is a constant. The case of interest is when the number of the required measurements is much smaller than the length of the signal. If the measurement vector is denoted as $y$, then we can write:

$$
y_{M \mathrm{x} 1}=\phi_{M \mathrm{x} N s} R_{N \mathrm{sx} 1},
$$

where $\phi$ is measurement matrix which is in our case defined by the positions of non-noisy signal samples. The Fourier basis matrix is used. From (8) and (12) follows:

$$
y=\phi R=\phi \psi \Re=\theta \Re \text {. }
$$

The system of equations defined by (13) consists of $M$ equations with $N_{s}$ unknowns. Therefore, the system is undetermined $\left(M<<N_{s}\right)$ and has infinite number of solutions. In order to obtain optimal solution, optimization algorithms are used. There are many optimization techniques. Each technique is based on finding the sparsest solution of (14). It is shown that optimal results are provided by using optimization techniques based on the $l_{1}$-minimization. Hence, the optimization problem is defined as:

$$
\widehat{\Re}=\min \|\Re\|_{l_{1}} \text { subject to } y=\theta \Re,
$$

where $\widehat{\Re}$ is a solution of the minimization problem, whereas the $l_{1}$-norm of vector $\mathfrak{R}$ is defined by:

$$
\|\Re\|_{l_{1}}=\sum_{i=1}^{N}\left|\Re_{i}\right| \text {. }
$$

The minimization problem is solved using the primal-dual basis pursuit method [27]. As a result we obtain the Fourier transform vector $\Re$ of local complex-lag auto-correlation coefficients $R$. For each time instant we obtain one vector $\Re$, which the resulting high quality distribution is called the CS based complex-lag distribution.

\section{NUMERICAL EXAMPLES}

In order to illustrate the efficiency of the proposed approach, let us consider the signal in the form: $x(t)=e^{j 150 \pi t^{4}+j 60 \pi t^{2}}+v(t)$, where $v$ is the impulse noise. The complex-time distribution is calculated for the original (non-noisy signal) and for the noisy signal. Further, the Lestimate form of the complex-time distribution is calculated (60\% of strongest samples are discarded and among them the noisy samples). Finally, the proposed CS based complex-time distribution is applied. The results are shown in Fig. 1, for a single time instant. It is obvious that the distribution calculated using noisy samples is useless for the analysis, Fig 1.b. The Lestimate form of the CTD (LCTD) is given in Fig 1.c, where new noisy effect appears as a consequence of missing samples. Finally, the CS based CTD is shown in Fig 1.d. Furthermore, the L-estimate form of the complex-time distribution (for all time instants) is given in Fig 2.a, while the proposed form is shown in Fig 2.b. It can be observed that the proposed CS based complex-time distribution significantly improves the results of the L-estimate form (Fig. 2b).
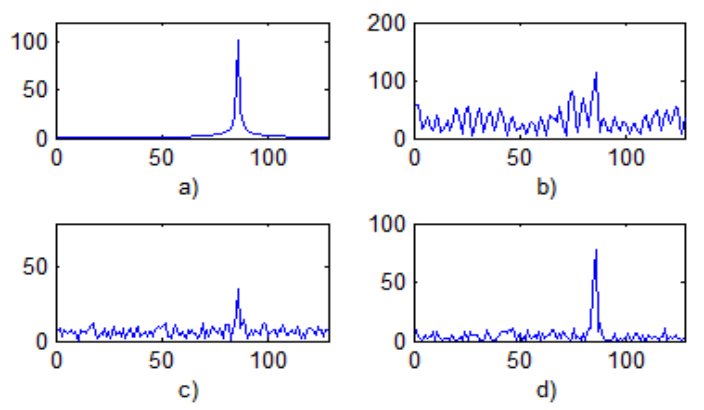

Fig 1. a) standard $\mathrm{CTD}_{4}$ of non-noisy signal, b) standard $\mathrm{CTD}_{4}$ of noisy signal, c) L-estimate form of the $\mathrm{CTD}_{4}$, d) $\mathrm{CS}$ based $\mathrm{CTD}_{4}$ 


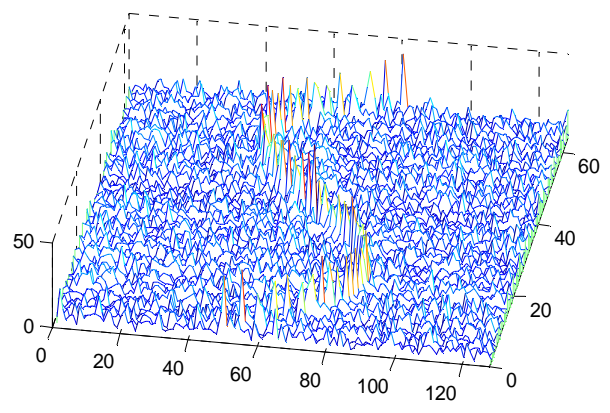

a)

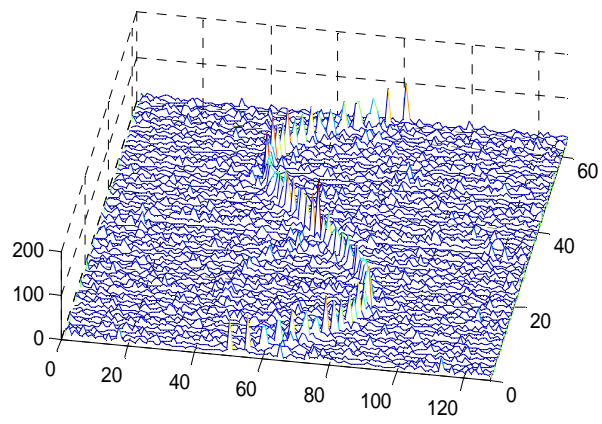

b)

Fig 2. a) L-estimate $\mathrm{CTD}_{4}$, b) Proposed $\mathrm{CS} \mathrm{CTD}_{4}$

\section{CONCLUSION}

Compressive sensing is applied to reconstruct the missing complex-lag autocorrelation samples, which appear as a consequence of L-estimation approach in noisy conditions. The L-estimation removes the impulse noise by omitting the noisy samples, but in the same time produces negative effects which can be seen as additional noise in the time-frequency domain. It has been shown that CS can effectively reconstruct missing samples of the complex-lag autocorrelation function, which leads to the highly concentrated spectral representation.

\section{REFERENCES}

[1] B. Boashash, "Estimating and interpreting the instantaneous frequency of a signal - Part 1," IEEE Proc., vol. 80, no.4, April 1992, pp. 519-538.

[2] B. Boashash, "Time-Frequency Analysis and Processing," Elsevier, 2003, Amsterdam

[3] J. Lerga, V. Sucic, "Nonlinear IF Estimation Based on the Pseudo WVD Adapted Using the Improved Sliding Pairwise ICI Rule," IEEE SP Letters, vol.16, no.8, pp. 953-956, 2009.

[4] E. Sejdic and J. Jiang, "'Time-Frequency Analysis of the Heart Sounds," in Proc. of 2002 ECEGRS, Ontario, Canada, May 15-17, 2002, pp. 5-9.

[5] F. Ahmad, M. G. Amin, "Through-the-wall human motion indication using sparsity-driven change detection," IEEE Trans. GRS, In Press

[6] I. Orovic, S. Stankovic, M. Amin, "A New Approach for Classification of Human Gait Based on Time-Frequency Feature Representations," Sig. Proc., vol. 91, no. 6, pp. 1448-1456, 2011.

[7] S. Stankovic, I. Djurovic, "Motion parameter estimation by using time frequency representations," El. Lett., vol.37, no.24, pp.1446-1448, 2001
[8] B.G. Mobasseri, "Digital Watermarking in joint time-frequency domain," IEEE International Conference on Image Processing, Rochester, NY, September 22-25, 2002.

[9] S. Stankovic, I. Orovic, N. Zaric, "An Application of Multidimensional Time-Frequency Analysis as a base for the Unified Watermarking Approach,"IEEE Trans on Image Proc, vol. 1, no. 3, 2010., pp.736-745

[10] L. Lee and S. Krishnan, "Time-frequency signal synthesis and its application in multimedia watermark detection," EURASIP Journal on Applied Signal Processing, Vol. 2006 (2006), Article ID 86712

[11] S. Stankovic, I. Djurovic, V. Vukovic, "System architecture for spacefrequency image analysis," El. Lett, vol.34, no.23, pp.2224-2245,1998

[12] X. Li, G. Bi, S. Stankovic, A. M. Zoubir, "Local polynomial Fourier transform: A review on recent developments and applications," Signal Processing, Vol. 91, No. 6, pp. 1370-1393, June, 2011

[13] B. Ristic, B. Boashash, "Relationships between the Polynomial and Higher order Wigner-Ville Distribution," IEEE Signal Processing Letters, vol. 2, no. 12, Dec. 1995, pp. 227-229.

[14] M. Morelande, B. Senadji, B. Boashash: "Complex-lag Polynomial Wigner-Ville distribution," IEEE TENCON, 1997, pp. 43-46.

[15] S. Stanković, N. Žarić, I. Orović, C. Ioana, "General form of timefrequency distribution with complex-lag argument," El. Lett., vol. 44 no. 11, pp. 699-701, 2008.

[16] S. Stanković, I. Orović, C. Ioana, "Effects of Cauchy Integral Formula Discretization on the Precision of IF Estimation: Unified Approach to Complex-Lag Distribution and its Counterpart L-Form," IEEE Signal Processing Letters, vol. 16, no. 4, April 2009., pp. 327-330.

[17] I. Orović, S. Stanković, "A Class of Highly Concentrated TimeFrequency Distributions Based on the Ambiguity Domain Representation and Complex-Lag Moment.," EURASIP Journal on Advances in Signal Processing, vol. 2009., Article ID 935314, 9 pages

[18] I. Orovic, M. Orlandic, S. Stankovic, Z. Uskokovic, "A Virtual Instrument for Time-Frequency Analysis of Signals with Highly NonStationary Instantaneous Frequency," IEEE Transactions on Instrumentation and Measurements, vol. 60, no. 3, pp. 791 - 803,2011

[19] R. Wang, Y. Jiang, "ISAR Imaging of Ship Based on the Modified 4thOrder Time-Frequency Distributions with Complex-lag Argument", Int. Conf. on Remote Sens, Environ. and Transportation Eng., 2012

[20] S. Stankovic, "Time-Frequency Analysis and its Application in Digital Watermarking," EURASIP Journal on Advances in Signal Processing, Special Issue on Time-Frequency Analysis and its Application to Multimedia signals, ID 579295, 20 pages, 2010

[21] N. Zaric, I. Orovic, S. Stankovic, "Hardware realization of generalized time-frequency distribution with complex-lag argument," EURASIP Journal on Advances in Signal Processing, ID 250794, 17 pages, 2009

[22] A. Omidvarnia, G. Azemi, J.O' Toole, B. Boashash, "Robust estimation of highly-varying nonlinear instantaneous frequency in monocomponent nonstationary signals," Signal Processing,04/ 2013

[23] R. Wang, Y. Jiang, "A modified fourth-order time-frequency distribution with complex-lag argument and its counterpart L-form", Int. Conf. on Consumers Electronics, Comm. and Networks., pp. 1531-1535

[24] S. Stankovic, I. Orovi, E. Sejdic, "Multimedia Signals and Systems," Springer, 2012.

[25] LJ. Stankovic, S. Stankovic, "Wigner distribution of noisy signals," IEEE Trans. on Signal Processing, vol.41., no.2., 1993., pp.956-960.

[26] N. Zaric, I. Orovic, S. Stankovic, "Robust Time-Frequency Distributions with Complex-lag Argument," EURASIP Journal on Advances in Signal Processing, Vol. 2010, Article ID 879874, 10 pages, 2010

[27] R. Baraniuk, "Compressive sensing," IEEE SP Magazine, vol. 24, no. 4, pp. 118-121, 2007

[28] LJ. Stankovic, S. Stankovic, I. Orovic, M. Amin, "Robust TimeFrequency Analysis based on the L-estimation and Compressive Sensing," IEEE Signal Processing Letters, vol. 20, no. 5, pp. 499-502

[29] E. Candes, and J. Romberg: 'L1-magic : Recovery of Sparse Signals via Convex Programming" http://www.acm.caltech.edu/l1 magic $\wedge \#$ code, 2005, 1-19 